2020-07

\title{
Weathering and persistence of plastic in the marine environment: Lessons from LEGO
}

Turner, Andrew

http://hdl.handle.net/10026.1/15560

10.1016/j.envpol.2020.114299

Environmental Pollution

Elsevier BV

All content in PEARL is protected by copyright law. Author manuscripts are made available in accordance with publisher policies. Please cite only the published version using the details provided on the item record or document. In the absence of an open licence (e.g. Creative Commons), permissions for further reuse of content should be sought from the publisher or author. 
1 Weathering and persistence of plastic in the marine

2 environment: Lessons from LEGO

3

4 Andrew Turner*1, Rob Arnold ${ }^{2}$, Tracey Williams ${ }^{3}$

$5{ }^{1}$ School of Geography, Earth and Environmental Sciences,

6 University of Plymouth

7 Drake Circus

8 Plymouth PL4 8AA, UK

9 aturner@plymouth.ac.uk

10

$11 \quad{ }^{2}$ Rame Peninsula Beach Care

1256 Fore Street

13 Kingsand

14 Torpoint PL10 1NA, UK

15

$16{ }^{3}$ The Lego Lost At Sea Project

17 Old Bridge House

18 Porth Bean Road

19 Newquay TR7 3LU, UK

20

21 Accepted $28^{\text {th }}$ February 2020

22 doi.org/10.1016/j.envpol.2020.114299

23 
The residence times of plastics in the oceans are unknown, largely because of the durability of the material and the relatively short (decadal) period of time over which plastic products have been manufactured. In this study, classic LEGO bricks constructed of acrylonitrile butadiene styrene (ABS) and washed up on the strandlines of beaches of southwest England have been subjected to X-ray fluorescence (XRF) analysis and the spectra and any other identifiers matched with unweathered blocks stored in collections or sets of known history. Relative to unweathered equivalents, weathered blocks exhibit varying degrees of yellowing, fracturing and fouling, and are of lower mass, average stud height and mechanical strength. These effects are attributed to photo-oxidative degradation and the actions of physical stress and abrasion while exposed to the marine environment. Infrared spectra indicate that the polymer remains largely intact on weathering but with photo-degradation of the polybutadiene phase of ABS, while quantification of XRF spectra reveals that pigments like cadmium sulphoselenide become more heterogeneously distributed in the matrix when in the environment. Using measured mass loss of paired (weathered versus unweathered) equivalents and the age of blocks obtained from storage we estimate residence times of between about 100 and 1300 years for this type and thickness of plastic, with variations reflecting differences in precise additive composition and modes of weathering.

Capsule: The weathering and persistence of plastic at sea has been studied by comparing LEGO blocks washed up on beaches with archived LEGO blocks of a similar age.

Keywords: LEGO; plastic; acrylonitrile butadiene styrene; marine; weathering; residence times

\section{Introduction}

Marine plastic has a variety of well-established impacts in the environment and on wildlife (Sheavy and Register, 2007; Barnes et al., 2009; Wilcox et al., 2016). Many of these impacts are highly pervasive and result from the exceptional durability of the material, or the very properties that make it such a versatile and widely-used commodity. Although plastic in the ocean is persistent, it will slowly weather through photo-oxidative degradation of the polymer and physical stress on the material (by, for example, abrasion and impaction). Markers of abiotic plastic degradation include a reduction in average molecular weight of the polymer, loss of mechanical properties, changes in 
surface characteristics (e.g. colour, texture) and shifts in spectral signatures (Andrady, 2015), with the latter characteristic forming the basis of most mechanistic and quantitative studies (Cooper and Corcoran, 2010; Turner and Holmes, 2011; Zhu et al., 2019).

The precise rates of plastic weathering in the marine environment are difficult to ascertain because the material has only been manufactured over a relatively short (decadal) time period and the original compositions and properties of polymers vary considerably (Ter Halle et al., 2017). Moreover, empirical studies conducted in the laboratory or field, where material is exposed to natural or simulated sunlight, and with or without abrasive particulates, have been restricted to timeframes of a few weeks to a few years (Da Costa et al., 2018; Brandon et al., 2016; Biber et al., 2019). An alternative, indirect means of studying plastic weathering and persistence and estimating age of deposition over a longer period is to consider negatively buoyant items with a distinct temporal source. For example, loakeimidis et al. (2016) compared clear polyethylene terephthalate (PET) bottles trawled from the bed of the Saronikos Gulf, Greece, with a newly purchased PET bottle, and were able to relate shifts in spectral band intensities from Fourier Transform Infrared (FTIR) spectrometry with expiration dates spanning a period of nearly two decades. Assuming that expiration dates are a proxy for time of deposition, the authors concluded that PET remains intact for approximately fifteen years, whereafter there was a significant decrease in the occurrence of native functional groups indicating chemical changes.

Over the past decade, voluntary organisations from Cornwall, southwest England, have retrieved plastic waste from beach cleans throughout the county, with items of interest archived. Among these items are blocks of classic LEGO, identifiable from distinct studs and tubes and, in many cases, specific design numbers within the interior structure. Since 1966, European LEGO has been constructed of acrylonitrile butadiene styrene (ABS), an amorphous, thermoplastic copolymer made of an acrylonitrile-styrene continuous phase and partially grafted polybutadiene that acts as an impact modifier (Saviello et al., 2014). The density of ABS ranges from about 1 to $1.2 \mathrm{~g} \mathrm{~cm}^{-3}$ depending on processing method, performance requirements (or relative abundance of the three monomers) and the type and amount of functional additives present (Mitchell, 1996). Regarding LEGO, additives have evolved over the years because of changes in material costs, production locations, technology and environmental regulations. Of particular significance in respect of the latter is the use of the now restricted brightly-coloured yellow and red cadmium-based pigments from the early 1970 s to the early 1980s (Turner, 2018).

In the present study, samples of classic LEGO blocks temporarily washed up on the strandlines of beaches of southwest England have been matched with blocks in $\sim 40+$ year-old collections or sets 
that have not undergone environmental exposure from similarities in their secondary (fluorescent) X-ray spectra. Weathered and unweathered sample pairs are quantitatively and qualitatively compared using a variety of techniques in order to examine the physical and chemical changes and rates of weathering effected by exposure to the marine environment over decadal timeframes.

\section{Materials and Methods}

About 50 beached LEGO blocks (bricks, plates, tiles) were supplied to the university from archived litter collections (dating back to 2010) held by various voluntary organisations engaged in regular beach cleans in Cornwall, the most southwesterly county in England that is bordered by the western English Channel to the south and the southern Celtic Sea to the north. In theory, blocks are denser than coastal sea water $\left(\sim 1.02 \mathrm{~g} \mathrm{~cm}^{-3}\right)$ and should sink, but plastic of this nature appears to be beached in Cornwall under certain tidal and meteorological conditions. In particular, relatively dense plastics can be found amongst large deposits of kelp washed up after strong onshore winds and large swell.

In the laboratory, LEGO blocks were rinsed under tap water and as much visible adherent or trapped extraneous material as possible (mainly sand and grit) was removed with a Nylon brush or metal tweezers. Samples were air-dried at $40^{\circ} \mathrm{C}$ for an hour before being individually weighed on a fivefigure Sartorius Genius balance; the length, width and height of each block and the height of all or at least three studs through their centres were then determined to $\pm 10 \mu \mathrm{m}$ with Allendale digital callipers. Density was estimated from the mass of tap water displaced by each block when suspended on a steel wire attached to the roof panel of the balance.

The chemical characteristics of each block were determined at between 4 and 12 locations by a battery-operated, energy-dispersive X-ray fluorescence (XRF) spectrometer (Niton XL3t He GOLDD+; Turner and Solman, 2016). The instrument was housed in an accessory stand with the nose pointing upwards, and the measurement surface was suspended just above the 8-mm diameter detector window. Counting was conducted in a plastics mode with a thickness correction of $1.5 \mathrm{~mm}$ (the approximate thickness of most blocks) for 20 seconds at $40 \mu \mathrm{A}$ and $50 \mathrm{kVp}$ and 10 seconds at $100 \mu \mathrm{A}$ and $20 \mathrm{kVp}$.

Blocks of the same (or original) colour, structure and size and, if available, serial number or internal signage, that had been boxed while not in use and that were in good condition ("unweathered") were sourced from collections purchased in the UK between 1972 and 1981. Samples were subjected to XRF analysis as above and those whose spectra and pigment composition most closely 
matched beached ("weathered") blocks were paired. Particular attention was paid to compounds of

$121 \mathrm{Cd}$ as these were employed over a specific timeframe that is difficult to define precisely but appears to have been from the early 1970s until their replacement on health and environmental grounds at the beginning of the following decade (Compound Interest, 2019). Specifically, high quantities of CdS and $\mathrm{CdS}_{1-x} \mathrm{Se}_{x}(0 \leq x \leq 1)$ were employed as bright yellow and red pigments, respectively, while unknown $\mathrm{Cd}$ compounds appear to have been added at lower concentrations to blocks of other colours but whose function is unclear (Turner, 2018). Examples of paired sample spectra are illustrated in Figure 1 for red bricks with common characteristic peaks for $\mathrm{Cd}$, Se and Ba (sample 1) and for white bricks with common peaks for Ti but without $\mathrm{Zn}$ that was present in many other white LEGO blocks (sample 4). Unweathered samples were measured for mass, size, stud height and density as above and were retained for comparative purposes and further characterisation.

Weathered and unweathered LEGO blocks were analysed by attenuated total reflectance Fouriertransform-infrared (ATR-FTIR) spectrometry using a Bruker Vertex 70. A stainless steel scalpel was used to scrape a piece of a few $\mathrm{mg}$ from different regions of the sample surface to a depth of about $100 \mu \mathrm{m}$. Scrapings were clamped with the outer face against the diamond crystal before spectra were acquired with 16 scans in the region 4000 to $600 \mathrm{~cm}^{-1}$ and at a resolution of $4 \mathrm{~cm}^{-1}$. The surfaces of selected weathered and unweathered blocks were photographed under a Nikon SMZ800 stereo-microscope, while the surface morphology and surficial elemental composition of these blocks were compared by scanning electron microscopy (SEM) using a JEOL JSM-6610. The electron microscope was operated with an accelerating voltage of $15 \mathrm{kV}$ and in a low vacuum mode, and was outfitted with an Oxford Instruments energy dispersive X-ray spectrometer (EDS) and Aztec software. The mechanical strength of selected weathered and unweathered blocks was determined in compression tests using an Instron 5582 Universal Tester operated at a crosshead speed of $2 \mathrm{~mm}$ $\mathrm{min}^{-1}$ and with load-displacement data monitored and recorded in Merlin software.

\section{Results and Discussion}

\subsection{Visual and physical changes of LEGO blocks on weathering}

147 The blocks subject to weathering in the marine environment that are considered in the study are 148 shown in Figure 2 alongside corresponding unweathered blocks that were paired in terms of age and 149 origin from similarities in XRF spectra. Thus, a total of 14 pairs were identified, with the majority 150 comprising classic bricks of 2, 4 or 8 studs; remaining pairs consisted of a sloping (roof) brick (sample 151 8), a brick with a moulded axel housing (sample 6), a 2-studded plate (sample 13) and a 1-studded 
cylinder (sample 14). Weathered blocks have smoother edges and corners compared with unweathered equivalents, and their glossy sheen is lost and the embossed LEGO logo on the studs and any designed textures have disappeared. While the colour of black, grey and green blocks remain on weathering, red, yellow and blue blocks appear to fade or haze and all red blocks and one white block exhibit evidence of "yellowing". Aside from smoothing and denudation, many weathered blocks reveal structural deformities, including distortion or contraction, and evidence of fracturing and fragmentation, with pits, grooves or gaps along the side or top face and missing connecting pins or cracked tubes in the interior. Overall, five weathered bricks were sufficiently fractured that significant parts of the structure (at least $3 \mathrm{~mm}$ in the longest dimension) were absent, and in two of these cases there were visible accumulations of biofouling, including calcareous deposits from what appeared to be the tube-building worm, Spirobranchus triqueter, within the internal structure.

Measurements of length, width and thickness did not vary systematically among paired samples and could not be used to evaluate the degree of block weathering; for example, in some cases measured dimensions increased upon weathering because of distortion of part or all of the plastic structure (compare, for example, block shapes in paired sample 7 in Figure 2). Height, however, and in particular stud height, appeared to provide a more robust evaluation of the extent of sample weathering as and shown in Table 1. Here, the mean height of studs on unweathered blocks ranges from about 1.7 to $1.9 \mathrm{~mm}$ while on weathered blocks mean height ranges from 0.5 to $1.8 \mathrm{~mm}$, and in all but one case where multiple measurements were taken there is a statistically significant reduction in stud height $(p<0.05)$ on weathering according to a series of two-sample $t$-tests. Overall, stud height is reduced significantly by between about $8 \%$ and over $60 \%$ on block weathering.

Also shown in Table 1 are the masses of unweathered and weathered sample pairs. Here, unweathered mass is given as a single value but where replicate matching blocks were available mass varied by less than $1 \%$. Mass reduction on weathering ranges from about $3 \%$ to $40 \%$ but this measure did not correlate with stud height reduction because the greatest losses in mass were accompanied by absence (through fracturing-disintegration) of significant parts of the LEGO block structure itself. Moreover, in some cases mass reduction was partially offset by visible fouling or entrapment of particulates that could not be shifted on cleaning.

All but three unweathered blocks sank in tap water and estimates of density derived from water mass displacement ranged from 1.00 to $1.17 \mathrm{~g} \mathrm{~cm}^{-3}$. Overall, however, there were no clear density differences between weathered and unweathered blocks, with colour, and presumably additive 
composition, having a more important effect on the results (grey and white blocks were densest and green and black blocks least dense).

Results of the compression tests are exemplified for two sample pairs in Figure 3. Here, the left and right peaks in the load-displacement distributions are associated with the compression of studs into the top wall and yielding of the block, respectively, with the maximum load in the latter peak indicative of ultimate compressive strength. Subsequent irregularities in the distributions, that are particularly apparent in weathered block 3 , are indicative of multiple failures taking place. Overall, we may conclude that weathered blocks are mechanically weaker and more brittle than their unweathered counterparts.

\subsection{Microscopy and chemical effects}

Microscopic images of the studded areas of two sample pairs, shown in Figure 4, illustrate the scale and heterogeneity of the aforementioned effects of weathering on the LEGO surface more clearly. In addition, comparative images reveal the extent of white bio-deposits on the surface of some weathered blocks and the propensity for cracks and pits to trap grains of sand and other microscopic debris. Images of greater magnification derived from SEM are exemplified for sample pair 10 in Figure 5. For the unweathered brick, the surface is relatively flat and uniform, and EDS results probing the upper few tens of microns of the sample are consistent with the composition of the polymeric matrix and the presence of (i) oxygen as oxidised products or as a component of inorganic additives, (ii) a Ba-based additive (that appears to be restricted to the light-coloured areas) and (iii) the bright yellow pigment, cadmium sulphide. The weathered brick is more heterogeneous in appearance, with microscopic cracks, notches, pores, pits and grooves distributed across the surface, and the EDS results indicate the presence of residual seawater salts and both inorganic (e.g. Al, Fe) and biological (e.g. $\mathrm{Ca}$, Si) fouling that obscure the chemical characteristics of the underlying plastic.

A more accurate but less sensitive evaluation of the concentration of a smaller range of elements in the samples was obtained by XRF spectrometry that employs a higher energy X-ray beam. Specifically, concentrations averaged through the $1.5 \mathrm{~mm}$ thickness of ABS were acquired from fluorescent spectral peaks that had been subject to fundamental parameters calculations using Niton NDT software. In individual unweathered blocks, and where detected, the XRF returned similar concentrations of $\mathrm{Ba}, \mathrm{Cd}, \mathrm{Se}, \mathrm{Ti}$ and $\mathrm{Zn}$ over each surface, including studded areas, suggesting an homogenous distribution of pigments and other inorganic additives throughout the $A B S$, and that surface geometry does not exert a measurable impact on the results. For weathered blocks, 
elemental concentrations were more varied across each sample suggesting a heterogeneous dissociation and migration of additives from the plastic as well as variable rates of disintegration of the plastic matrix. This effect is exemplified in Figure 6 for concentrations of Se versus concentrations of $\mathrm{Cd}$ determined over multiple areas of the surface ( $n=4$ to 12 ) of three sample pairs where cadmium sulphoselenide was used for colour. Thus, individual weathered blocks exhibit a wider distribution of concentrations of Se or $\mathrm{Cd}$ (or both Se and $\mathrm{Cd}$ ) than corresponding concentrations returned for unweathered blocks, and the effect is most pronounced for paired sample 1 where the weathered brick exhibited considerable distortion, fracturing and fouling. Note that the gradient of the mass relationship defining Se and Cd in unweathered blocks (0.155) suggests that the structure of the original pigment in the ABS may be approximated as: $\mathrm{CdS}_{0.78} \mathrm{Se}_{0.22}$.

FTIR spectra of weathered and unweathered LEGO blocks are exemplified in Figure 7 for four sample pairs of different colours. The majority of main bands in both spectra are consistent with the principal absorbing groups of ABS (e.g. styrene, acrylonitrile and butadiene; Saviello et al., 2014), with spectral intensities similar in all cases with the exception of black bricks where infrared radiation is readily absorbed (Becker et al., 2017). Overall, therefore, there is no clear evidence of the presence of organic additives in the matrix and it appears that the polymer remains largely intact on weathering, possibly because of the protective effects afforded by fouling, certain oxidation products or coloured pigments (Iannuzzi et al., 2013). The most significant differences between weathered and unweathered blocks appear to be restricted to the accentuation of a peak at 1735 $\mathrm{cm}^{-1}$ and the appearance of a peak at $1270 \mathrm{~cm}^{-1}$ on weathering. The former is due to carbonyl stretching of an ester group formed by the photo-oxidation of the polybutadiene phase of ABS while the latter likely results from $\mathrm{C}-\mathrm{O}$ stretching in oxidation products containing, for example, carboxylic acids (Saviello et al., 2014).

\subsection{Rates and mechanisms of LEGO weathering}

Because weathered LEGO blocks washed up on and retrieved from beaches of southwest England over the past decade were successfully paired with unweathered equivalents that were manufactured and purchased between the early 1970s and early 1980s, we may surmise that beached samples have been exposed to the local marine environment for between 30 and 40 years. Precisely how these blocks entered the environment, however, is unclear. In 1997 a welldocumented spillage of specific sets of customised LEGO pieces occurred off the coast of south west England (Osgood and Robinson, 2019) but no earlier spillages have been reported in the media or literature and the samples studied here do not appear to belong to specific sets. It is possible that 
individual blocks are lost at play on the beach but research and calculations undertaken by one of the UK's largest insurance companies suggests that over two million blocks have been flushed down the toilet by children under ten years old (Direct Line Home Insurance, 2016). Depending on the presence, nature and performance of historical waste water treatment plants, an unknown proportion of blocks lost during the early 1970 s to early 1980 s will have entered the aquatic environment via this route.

In theory, LEGO ABS is inherently denser than seawater, suggesting that blocks have a propensity to sink in the marine environment. This was confirmed by the density measurements of all weathered and most unweathered blocks being above $1.03 \mathrm{~g} \mathrm{~cm}^{-3}$. There is, however, no means of ascertaining precisely how and where the LEGO blocks were weathered. For example, blocks may have been gradually degraded photolytically in seawater during suspension above or deposition on the seabed, an effect that is often constrained by the protective (e.g. shading) properties of surface fouling (Andrady, 2015); abrasive weathering by sediment and other particulates may have taken place during turbulence near or on the seabed (and particularly in the swash zone), and fracturing may have resulted from physical stress and impaction on hard surfaces. It is also significant that $\mathrm{Cd}$ sulphide and sulphoselenide pigments are light-sensitive (Fowles, 1977; Liu et al., 2017) because we may surmise that pigment release as well as polymer breakdown are accelerated in the photic zone. This assertion is consistent with the presence of visible calcareous biodeposits on the most weathered red and yellow cadmium-pigmented blocks.

Regardless of the precise mechanisms of LEGO block weathering, an evaluation of environmental persistence may be gained from the mass losses reported in Table 1 and the aforementioned estimates of periods of exposure. Thus, neglecting any fouling and accumulation of material, net block mass loss ranges from about $3 \%$ to $40 \%$, suggesting that this type and thickness of plastic may remain in the marine environment for between about 100 and 1300 years. Presumably, upper estimates reflect gradual degradation, possibly where light is limited, and lower estimates involve additional physical stress and subsequent fragmentation. These estimates compare with life expectancies of clear polyethylene terephthalate bottles (whose thickness is typically a few hundred $\mu \mathrm{m}$ ) of between several tens and one hundred years (loakeimidis et al., 2016) and are consistent with the assertion that the residence times of plastics in the ocean are believed to exceed the timeframes over which the materials have been manufactured (Barnes et al., 2009).

\section{Conclusions}


In summary, analysis of paired weathered-unweathered LEGO blocks has allowed the physical and chemical effects of marine environmental exposure on pigmented ABS plastic to be examined.

Weathering over decadal timeframes results in smoothing, discolouration and fouling of the plastic surface and deformation, fracturing and fragmentation of the structure, with leaching of additives and pigments from the matrix proceeding at variable rates. Based on mass difference among paired samples that are about 40 years old we estimate residence times in the marine environment on the order of hundreds of years.

\section{Acknowledgements}

The authors a grateful for the technical support provided by Mr Billy Simmonds and Mr Glenn Harper, Plymouth University. The study was funded partly by a Plymouth Marine Institute HEIF Award.

\section{References}

Andrady, A.L., 2015. Persistence of plastic litter in the oceans. In: Marine Anthropogenic Litter, ed. M. Bergmann, L. Gutlow, M. Klages. Springer, pp. 57-72.

Barnes, D.K.A., Galgani, F., Thompson, R.C., Barlaz, M., 2009. Accumulation and fragmentation of plastic debris in global environments. Philosophical Transaction of the Royal Society of London: B Biological Sciences 364, 1985-1998.

Becker, W., Sachsenheimer, K., Klemenz, M., 2017. Detection of black plastics in the middle infrared spectrum (MIR) using photon up-conversion technique for polymer recycling purposes. Polymers 9 , 435 doi:10.3390/polym9090435

Biber, N.F.A., Foggo, A., Thompson, R.C., 2019. Characterising the deterioration of different plastics in air and seawater. Marine Pollution Bulletin 141, 595-602.

Brandon, J., Goldstein, M., Ohman, M.D., 2016. Long-term aging and degradation of microplastic particles: Comparing in situ oceanic and experimental weathering patterns. Marine Pollution Bulletin 110, 299-308.

Compound Interest, 2019. What are LEGO bricks made of? https://www.compoundchem.com/2018/04/09/lego/ accessed 8/19

Cooper, D.A., Corcoran, P.L., 2010. Effects of mechanical and chemical processes on the degradation of plastic beach debris on the island of Kauai, Hawaii. Marine Pollution Bulletin 60, 650-654.

Da Costa, J.P., Nunes, A.R., Santos, P.S.M., Girao, A.V., Duarte, A.C., Rocha-Santos, T., 2018.

Degradation of polyethylene microplastics in seawater: Insights into the environmental degradation of polymers. Journal of Environmental Science and Health Part A: Toxic/Hazardous Substances and Environmental Engineering 53, 866-875. 
Direct Line Home Insurance, 2016. https://www.directline.com/media/archive-2016/millions-ofLego-bricks-get-flushed-down-the-toilet accessed 8/19.

Fowles, G.W.A., 1977. The leaching of cadmium from plastic toys. Science of the Total Environment 7, 207-216.

Iannuzzi, G., Mattsson, B., Rigdahl, M., 2013. Color changes due to thermal ageing and artificial weathering of pigmented and textured ABS. Polymer Engineering and Science DOI 10.1002/pen.23438.

loakeimidis, C., Fotopoulou, K.N., Karapanagioti, H.K., Geraga, M., Zeri, C., Papathanassiou, E., Galgani, F., Papatheodorou, G., 2016. The degradation potential of PET bottles in the marine environment: An ATR-FTIR based approach. Scientific Reports 6:23501 DOI: 10.1038/srep23501.

Liu, H., Gao, H., Long, M., Fu, H., Alvarez, P.J.J., Li, Q., Zheng, S., Qu, X., Zhu, D., 2017. Sunlight promotes fast release of hazardous cadmium from widely-used commercial cadmium pigments. Environmental Science and Technology 51, 6877-6886.

Mitchell, P.E., 1996. Tool and Manufacturing Engineers Handbook, fourth edition. Vol. VIII: Plastic Part Manufacturing. Society of Manufacturing Engineers, Dearborn, Michigan.

Osgood, J., Robinson, K.H., (editors) 2019. Feminists Researching Gendered Childhoods: Generative Entanglements. Bloomsbury Press 192pp.

Saviello, D., Pouyet, E., Toniolo, L., Cotte, M., Nevin, A., 2014. Synchrotron-based FTIR microspectroscopy for the mapping of photo-oxidation and additives in acrylonitrile-butadienestyrene model samples and historical objects. Analytica Chimica Acta 843, 59-72.

Sheavy, S.B., Register, K.M., 2007. Marine debris and plastics: Environmental concerns, sources, impacts and solutions. Journal of Polymers and the Environment 15, 301-305.

Ter Halle, A., Ladriat, L., Martignac, M., Mingotaud, A.F., Boyron, O., Perez, E., 2017. To what extent are microplastics from the open ocean weathered? Environmental Pollution 227, 167-174.

Turner, 2018. Concentrations and migratabilities of hazardous elements in second-hand children's plastic toys. Environmental Science and Technology 52, 3110-3116.

Turner, A., Holmes, L., 2011. Occurrence, distribution and characteristics of beached plastic production pellets on the island of Malta (central Mediterranean). Marine Pollution Bulletin 62, 377381.

Turner, A., Solman, K.R., 2016. Analysis of the elemental composition of marine litter by fieldportable-XRF. Talanta 159, 262-271.

Wilcox, C., Mallos, N.J., Leonard, G.H., Rodriguez, A., Hardesty, B.D., 2016. Using expert elicitation to estimate the impacts of plastic pollution on marine wildlife. Marine Policy 65, 107-114.

Zhu, K.C., Jia, H.Z., Zhao, S., Xia, T.J., Guo, X.T., Wang, T.C., Zhu, L.Y., 2019. Formation of environmentally persistent free radicals on microplastic under light irradiation. Environmental Science and Technology 53, 8177-8186. 
Table 1: Mean stud height ( \pm one standard deviation) and mass for weathered and unweathered pairs of LEGO blocks, and the mean percentage reduction of stud height and mass on weathering. Asterisks denote a significant reduction in stud height on weathering according to a series of two-sample $t$-tests.

\begin{tabular}{|c|c|c|c|c|c|c|}
\hline \multirow[b]{2}{*}{ sample pair } & \multicolumn{2}{|l|}{ weathered } & \multicolumn{2}{|l|}{ unweathered } & \multicolumn{2}{|c|}{$\begin{array}{l}\text { reduction } \\
\text { on } \\
\text { weathering }\end{array}$} \\
\hline & stud height, $\mathrm{mm}$ & mass, $\mathrm{g}$ & $\begin{array}{c}\text { stud height, } \\
\mathrm{mm}\end{array}$ & mass, $\mathrm{g}$ & stud height, $\%$ & mass, $\%$ \\
\hline 1 & $1.13 \pm 0.10$ & 2.429 & $1.78 \pm 0.07$ & 3.248 & $36.2^{*}$ & 25.2 \\
\hline 2 & $1.49 \pm 0.09$ & 2.091 & $1.71 \pm 0.02$ & 2.216 & $13.1^{*}$ & 5.66 \\
\hline 3 & $1.11 \pm 0.29$ & 1.750 & $1.80 \pm 0.03$ & 2.179 & $38.4 *$ & 19.7 \\
\hline 4 & $1.64 \pm 0.05$ & 1.347 & $1.79 \pm 0.06$ & 1.467 & $8.40 *$ & 8.20 \\
\hline 5 & $1.67 \pm 0.10$ & 0.639 & $1.72 \pm 0.10$ & 0.831 & 2.91 & 23.1 \\
\hline 6 & $1.24 \pm 0.50$ & 1.229 & $1.84 \pm 0.05$ & 1.358 & $32.7^{*}$ & 9.40 \\
\hline 7 & $1.29 \pm 0.45$ & 1.139 & $1.92+0.05$ & 1.370 & $32.5^{*}$ & 16.9 \\
\hline 8 & $0.66 \pm 0.61$ & 1.250 & $1.94 \pm 0.05$ & 2.079 & $66.3^{*}$ & 39.8 \\
\hline 9 & $1.11 \pm 0.17$ & 0.908 & $1.74 \pm 0.07$ & 1.366 & $36.3^{*}$ & 31.6 \\
\hline 10 & $1.02 \pm 0.16$ & 2.356 & $1.72 \pm 0.03$ & 2.598 & $40.6^{*}$ & 9.31 \\
\hline 11 & $1.31 \pm 0.02$ & 1.465 & $1.78 \pm 0.04$ & 2.175 & $26.2^{*}$ & 32.6 \\
\hline 12 & $0.54 \pm 0.16$ & 1.034 & $1.69 \pm 0.07$ & 1.395 & $67.9 *$ & 25.8 \\
\hline 13 & $1.54 \pm 0.06$ & 0.349 & $1.88 \pm 0.04$ & 0.363 & $18.4^{*}$ & 3.91 \\
\hline 14 & 1.74 & 0.304 & 1.89 & 0.313 & 7.94 & 2.88 \\
\hline median & 1.27 & 1.2395 & 1.79 & 1.431 & 32.6 & 18.3 \\
\hline
\end{tabular}



2.

357
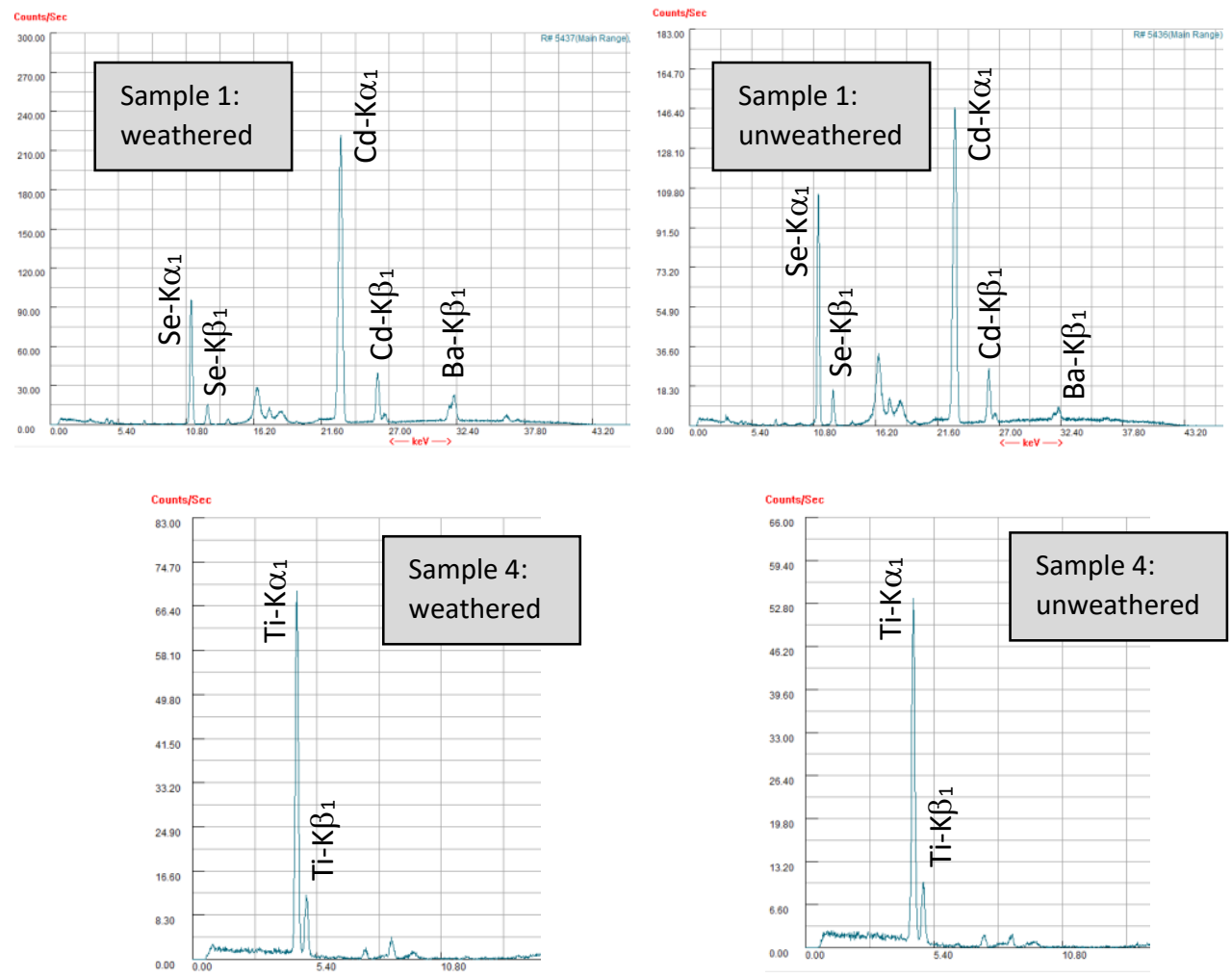
359

360

361

362

363

364

365

366

367

368

369

370

371

372

373

374

Figure 2: Weathered and unweathered blocks paired from appearance, signage and similarities in XRF spectra and shown on cm-scaled graph paper.

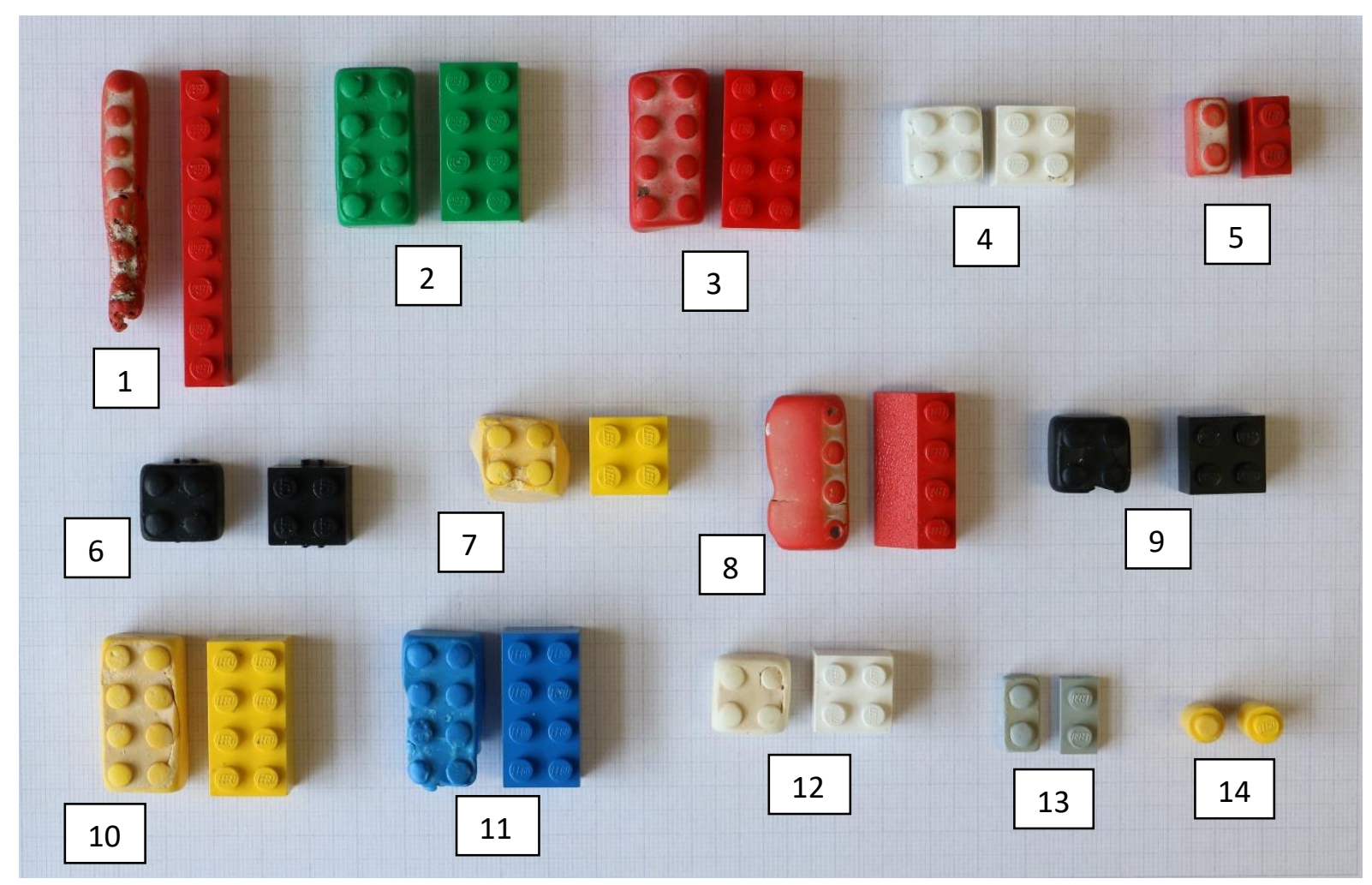


Figure 3: Load-displacement plots for unweathered blocks (solid lines) and weathered blocks (broken 376 lines).
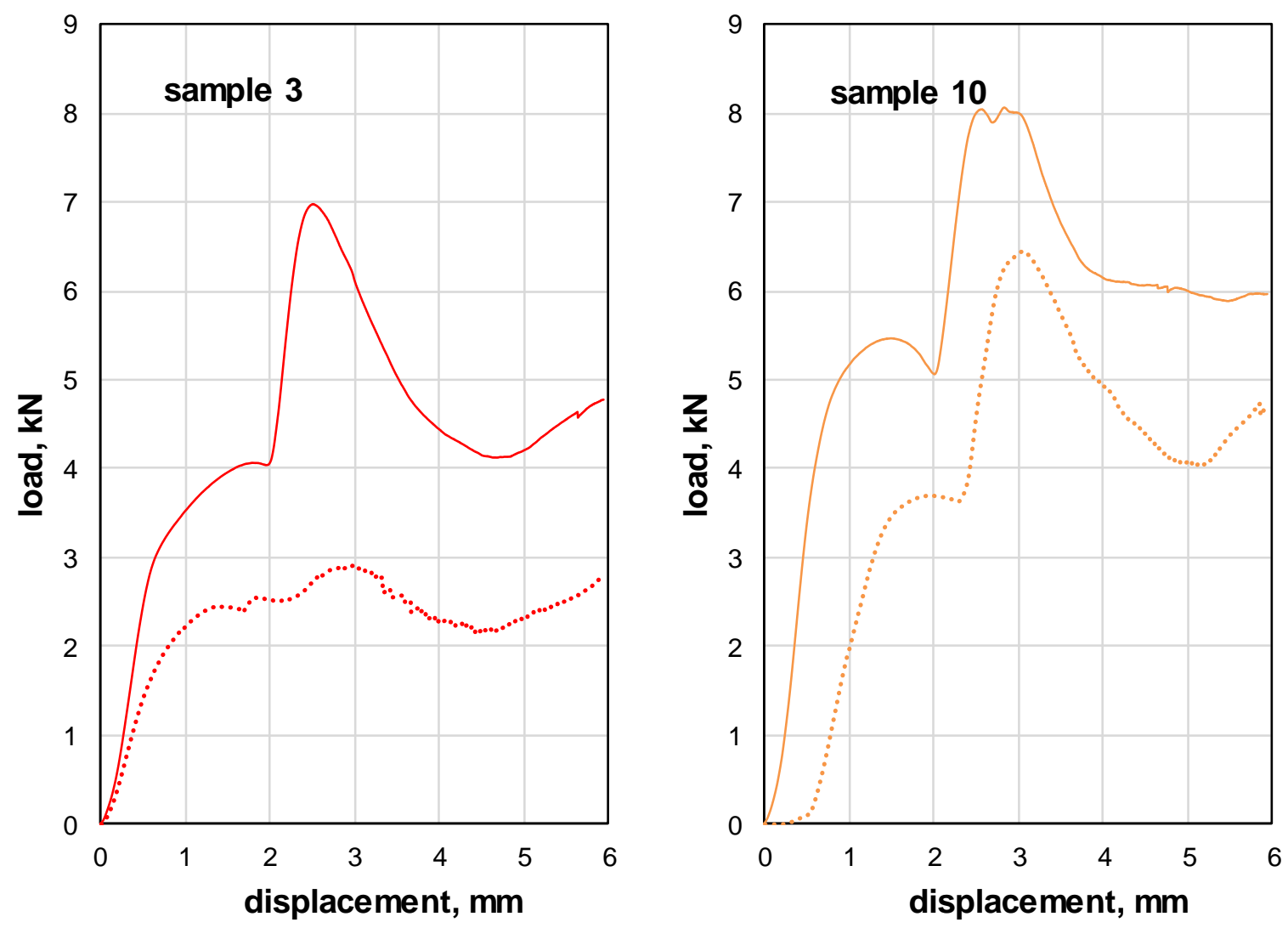

377

378

379

380

381

382 
383

384

385

386

387

388

389

390

391

392

Figure 4: Microscopic images of sample pairs 1 and 7. For scale, the diameter of the studs on the left are $4.85 \mathrm{~mm}$. Note the subtle change in font of the LEGO logo introduced in 1973.
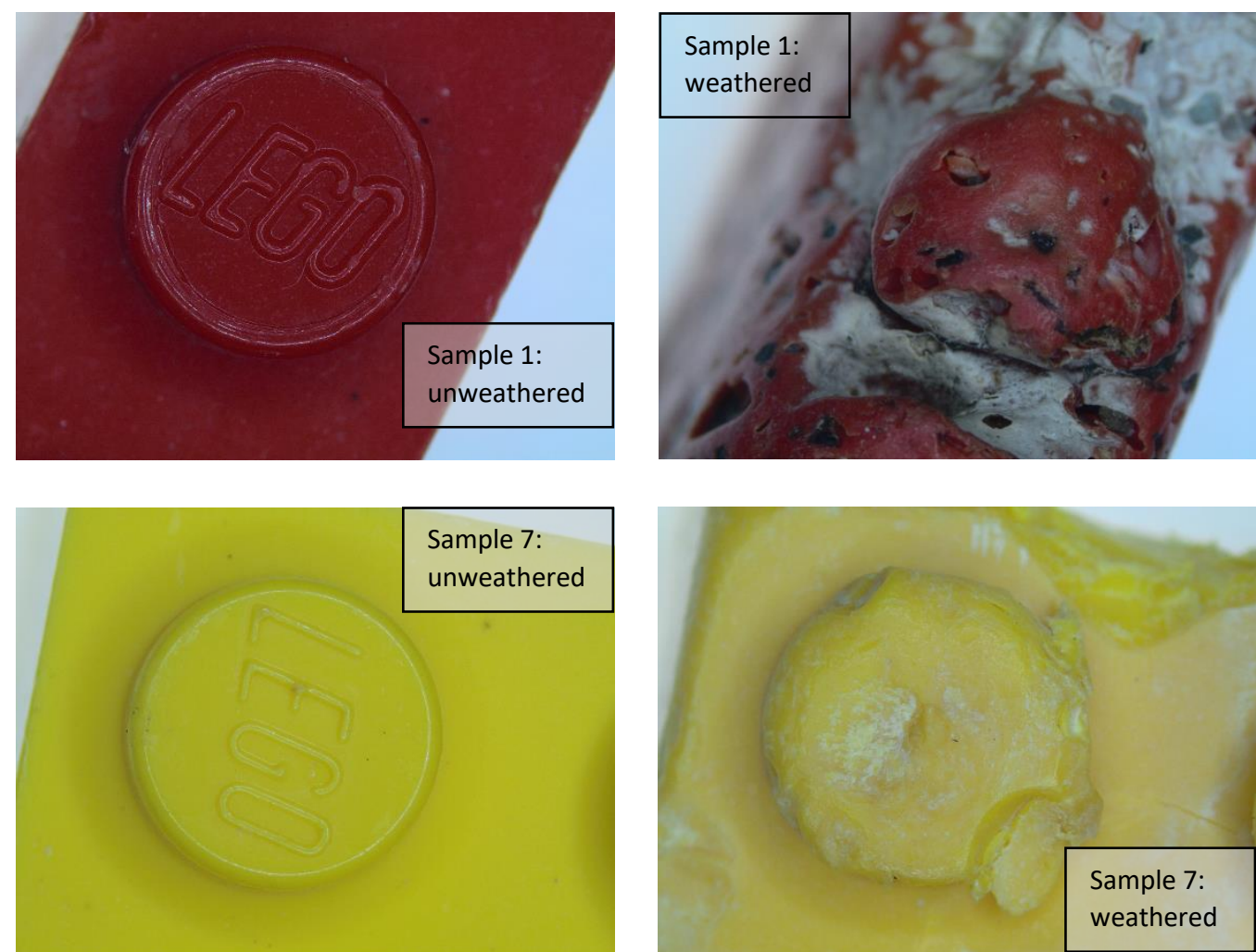
399

400

401

402

403

404

.
Figure 5: Electron microscope images of the shorter exterior wall of an unweathered and weathered LEGO block pair (sample 10) along with indicative elemental concentrations (in \%) derived at the locations shown circled in yellow. Note the presence of $\mathrm{Cd}$ as $\mathrm{CdS}$ in the unweathered block and the presence of $\mathrm{Ba}$ as an additive only in the lighter regions; in the weathered block these elements are absent at the surface because of the presence of residual salt and heterogeneous inorganic (e.g. Fe, Al) and organic (e.g. Si) fouling.

404
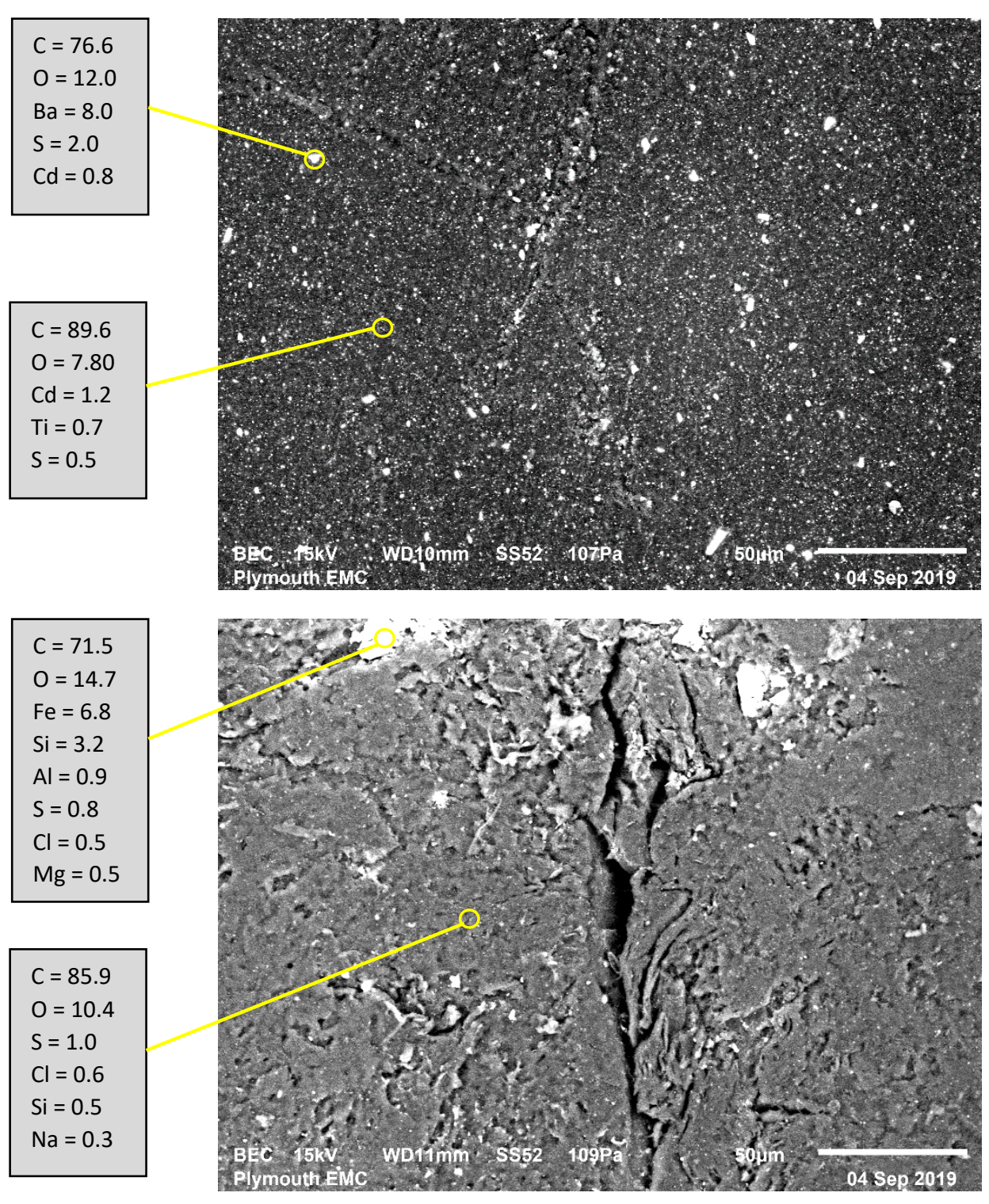
405

406

407

408

409

410

411

412

413

414

415

416

417

418

419

420

421

422

423

424

425
Figure 6: Concentrations of Se versus concentrations of $\mathrm{Cd}$ in three red samples pairs returned by multiple XRF measurements of each block. Sample $1=$ circles, sample $5=$ triangles, sample $8=$ squares; filled symbols = unweathered blocks and open symbols $=$ weathered blocks.

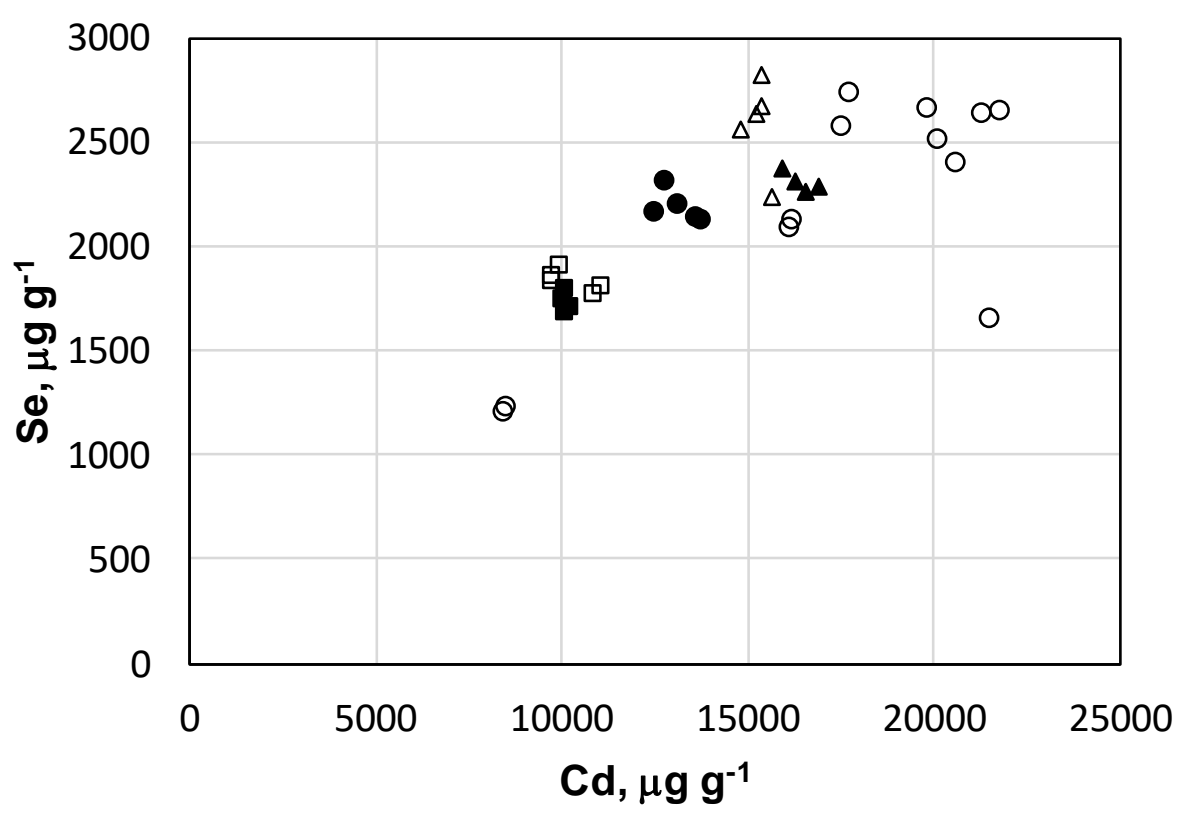


430

431

432

433

434

435

436

437

438

439
Figure 7: FTIR spectra between 700 and $4000 \mathrm{~cm}^{-1}$ for (a) unweathered and (b) weathered blocks (sample 8 = red; sample 9 = black; sample 10 = yellow; sample $12=$ blue). Note the accentuation of a peak at $1735 \mathrm{~cm}^{-1}$ and the appearance of a peak at around $1270 \mathrm{~cm}^{-1}$ in the weathered blocks.

29

31

32
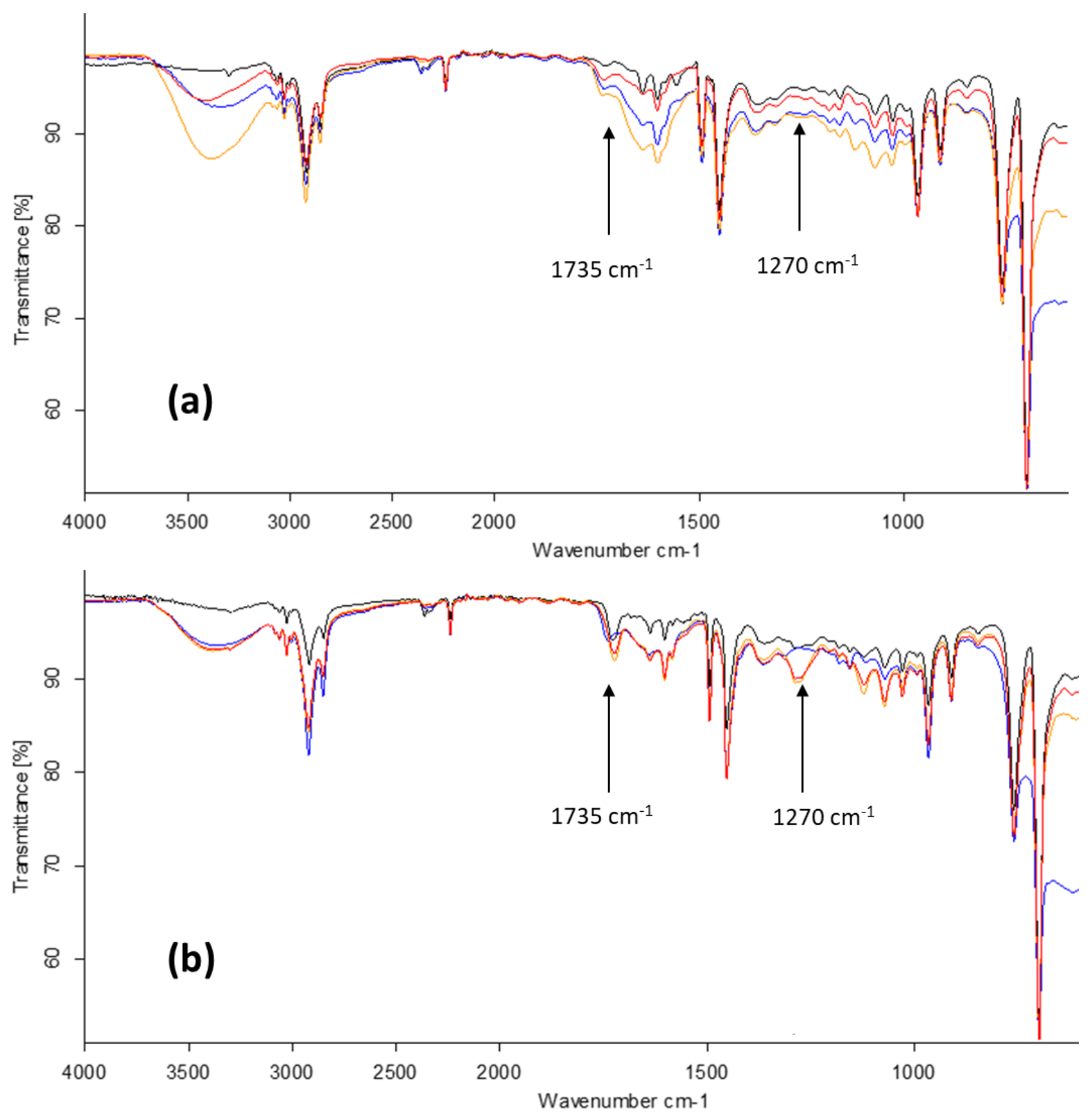